\title{
An Evidence-Based Vestibular Schwannoma Surgical Outcome Grading Scale for Large-to-Giant Vestibular Schwannomas
}

Nida Fatima ( $\square$ fnida99@yahoo.com )

House Clinic Inc: House Institute https://orcid.org/0000-0002-0976-888X

Gregory P. Lekovic

House Ear Institute: House Institute

\section{Research Article}

Keywords: Acoustic Neuroma, Acoustic tumor Grading System, Facial Nerve, Extent of Resection, Vestibular Schwannomas

Posted Date: December 20th, 2021

DOI: https://doi.org/10.21203/rs.3.rs-1167597/v1

License: (c) (i) This work is licensed under a Creative Commons Attribution 4.0 International License.

Read Full License 


\section{Abstract}

Objectives: The objective of this study was to determine the relationship between facial nerve function and extent of resection (EOR) as outcomes in the surgical management of large vestibular schwannoma (VS) ( $\geq 2.5 \mathrm{~cm}$ maximal) and evaluate use of a new grading system that incorporates both outcomes.

Methods: We conducted a systematic review of the electronic databases using different MeSH terms from 1990 to 2021. 5,623 patients from 56 studies were found appropriate for inclusion in the study. Surgical approach was reported in 5,144 patients, including translabyrinthine approach (TL) in 43.3\% $(n=2,225)$, retrosigmoid $(R S)$ approach in $56.3 \%(n=2,899)$, retrolabyrinthine $(R L)$ approach in $0.3 \%(n=16)$, and extended translabyrinthine (EX) approach in $0.1 \%(n=4)$. The proposed VS Grading System defines outcomes of gross total resection (GTR), near total resection (NTR) and subtotal resection (STR) with good facial nerve function [House-Brackmann (HB) Grade I-II] as Classes A, B and C respectively. Those with poor facial nerve outcome ( $\mathrm{HB} \mathrm{III}-\mathrm{VI})$ are graded as Classes $\mathrm{D}, \mathrm{E}$ and $\mathrm{F}$, respectively.

Results:. As expected, patients with STR had a higher likelihood of better facial nerve outcomes (HB I-II) compared to NTR [Odds Ratio (OR): 7.30, 95\%Cl: 2.45-12.1, $\mathrm{p}=0.004$ ] and GTR (OR: 9.61, 95\%Cl: 3.61-15.6, $\mathrm{p}=0.002$ ), while NTR had better facial nerve outcome than GTR (OR: 1.5, 95\%Cl: 1.14-4.6, $\mathrm{p}<0.0001)$. A Class A result, representing the best possible outcome, was obtained in $55.8 \%$ of TL vs. $49.4 \%$ undergoing RS approach.

Conclusion: Complete surgical resection with preservation of facial nerve function is the gold standard for large VS.

\section{Introduction}

Recently, the paradigm for vestibular schwannoma (VS) surgical treatment has been shifting towards what is sometimes referred to as a "nerve-centered" approach, in which preservation of facial nerve function (and, if possible, hearing) is the most important determinant of good versus poor outcome after surgery.[1-7] With the advancement in microsurgical techniques and neurophysiological monitoring, surgical mortality has been reduced to as low as $0.4 \%$, with anatomical preservation of facial nerve approaching $100 \%$. [8-10]

Although gross total resection (GTR) is the treatment of choice, near total resection (NTR) or subtotal resection (STR) is warranted in situations where facial nerve function is jeopardized by aggressive resection.[11] Therefore, there is a need to weigh the risk of future tumor growth against the benefit of facial nerve function in terms of extent of resection (EOR). The ideal EOR was historically controversial as Walter Dandy [12] opted for total resection of VS while Harvey Cushing [13] advocated partial resection to reduce morbidity. Further, William House reported in 1968 [14] that partial tumor removal was a 
reasonable alternative to total removal among patients who are elderly, have unstable vital signs, or are a poor surgical risk.

We aimed to address how to best evaluate surgical outcomes following resection of large to giant VS in particular. We believe, as do most other authors, that minimizing the risk of injury to facial nerve function is the paramount concern for the VS surgeon, and a post-operative facial nerve palsy is, generally speaking, incompatible with a good outcome overall. It follows that factors that expose the nerve to elevated risk should be avoided when possible. Studies have shown that EOR (usually categorized as GTR, NTR, and STR) is an independent risk factor for facial nerve injury, with the lowest risks of injury associated with STR in most studies [15-17].

What concerns us here is that EOR is most often treated as an independent risk factor for facial nerve outcome but not as a clinically relevant outcome in and of itself. In addition to the risk of facial nerve injury at the time of initial treatment, there is a risk of tumor recurrence with less than total removal, and, if severe enough, requirement for re-treatment with the consequent additional risk of facial or other nerve injury. Hence, the ideal surgical outcome is defined as that in which both risk of injury to facial nerve and tumor recurrence are minimized. Previous studies note that while STR is associated with good facial nerve outcome, it is also associated with higher tumor recurrence rates.[11],[18] Other studies have also shown that EOR is an independent risk factor for tumor recurrence.[11, 15-17] We contend that because EOR is an important proxy for the risk of recurrence, it should be considered as a surgical outcome, albeit secondary in importance to risk of facial nerve injury at the time of treatment.

We performed a systematic review and meta-analysis incorporating large, pooled data sets using double evaluation methods (coupling facial nerve outcome and EOR). The main determinant for good outcomes remains facial nerve function, but our proposed outcome scale distinguishes good surgical outcomes (good facial nerve function and STR) from better and best outcomes (good facial nerve function and NTR, vs GTR, respectively), as well as bad outcomes (GTR with poor facial nerve function) from "worse" and "worst" (NTR and STR with poor facial nerve function, respectively). Based on available data, this meta-analysis demonstrates that a surgical outcome grading scale using both facial nerve function and EOR as one integrated outcome may be useful for setting standard outcome reporting for patients undergoing VS surgery.

\section{Methods}

\section{Data search strategy:}

We followed the Preferred Reporting Items for Systematic Review and Meta-analysis (PRISMA) guidelines for literature search.[19] A detailed systematic review was performed of electronic databases using PubMed, Google Scholar, EMBASE, Medline, The Cochrane library, Scopus, CINAHL, Primary studies and FDA reports, bibliographies of the key articles, and ClinicalTrials.gov for articles published in the English 
language between January 1st, 1990 and August 1st, 2021.MeSH terms (using the Boolean operators "and" and "or") were used.

\section{Inclusion and Exclusion Criteria:}

The inclusion and exclusion criterion were based upon the PICOS framework which included, (i) Types of participants: Adult patients who underwent VS surgery for large to giant tumor $\geq 2.5 \mathrm{~cm}$, (ii) Interventions: Surgical treatment of VS using a variety of surgical approaches, (iii) Control: Not Applicable, (iv) Outcome measures: (a) Facial nerve function graded by the House-Brackmann (HB) scale at 1-year follow-up, (b) EOR (GTR, NTR and STR). Randomized controlled trials (RCTs), prospective cohort studies (PCS), retrospective comparative studies (RCS) and retrospective series were included in our analysis. Case reports and studies without a facial nerve outcome according to extent of resection were excluded. The study components along with inclusion and exclusion criterion are detailed in Table 1.

\section{Data Extraction and Outcome measures:}

The data were extracted using a structured template form based on the Cochrane Consumers and Communication Group. The meta-analysis was conducted in accordance with the criteria set by the Cochrane Consumers and Communication Group reviews: Meta-analysis. Both authors reviewed each article, and any disagreement among the authors was resolved by discussion. The following data were extracted from each article: (i) demographic characteristics, (ii) type of surgery (translabyrinthine, retrosigmoid, and others), (iii) extent of resection, and (iv) HB grade facial nerve outcome..

\section{Statistical analysis:}

Logistic regression analysis was used to measure the odds ratio (OR) and 95\% confidence interval (CI) using random effect model. All tests were 2-tailed, and p value $<0.05$ was considered statistically significant. SPSS 23 was used to conduct independent sample t-tests and Spearman's coefficient correlation. For the establishment and validation of new grading system, z-test for the difference between two proportions was carried out.

\section{Risk of bias across studies.}

The Cochrane Collaboration's tool was used to assess the risk of bias across the included studies, including symmetry of the funnel plots and the Egger test to evaluate publication bias. A non-parametric trim-and-fill procedure identified and corrected for funnel plot asymmetry and re-estimated the aggregate results. The high heterogeneity among studies was further analyzed using funnel plots, which showed asymmetrical distribution. This bias can be attributable to sample size as the removal of smaller-sized cohorts significantly decreased heterogeneity.

\section{Study Selection:}

A total of 335 articles were retrieved from the various electronic databases (see Search Strategy in Methods) and reviewed according to PRISMA guidelines. After screening the abstracts, 256 articles were excluded as the data were not related to surgical treatment of large VS or did not present quantitative 
results. A total of 79 full text articles were assessed for eligibility, of which 23 were excluded based upon the inclusion and exclusion criteria. The remaining 56 articles were included in this review, including nonRCTs [20]'[8, 15-17, 21-52, 52-71].

\section{Overall characteristics of the study:}

The 56 studies provided a total of 5,623 patients for analysis, of which complete data for surgical approach was available for 5,144 (91.4\%) patients. Baseline characteristics of the included studies are presented in Table 2. Surgical approaches included translabyrinthine in $43.3 \%(n=2,225 / 5,144)$, retrosigmoid in $56.3 \%(n=2,899 / 5,144)$, retrolabyrinthine in $0.3 \%(n=16 / 5,144)$, and extended translabyrinthine in $0.1 \%(n=4 / 5,144)$.

\section{Results}

Overall, the studies reported EOR in 4,533 (80.6\%) patients which included GTR in $83.2 \%$ of the patients $(n=3,775 / 4,533)$, NTR in 3.8\% $(n=167 / 4,533)$ and STR in 13.0\% $(n=591 / 4,533) .$. Good HB facial nerve function was reported among $56.8 \%(n=2,145 / 3,775)$ undergoing GTR, 85.3\% ( $n=111 / 130$, data missing for 37 patients) undergoing NTR, and $84.5 \%$ ( $n=469 / 555$, data missing for 36 patients) undergoing STR .

\section{Facial Nerve Outcome and Extent of Resection as Independent Variables:}

There is a higher likelihood of better facial nerve outcomes (HB I-II) compared to near total resection (OR: 7.30, 95\%Cl: 2.45-12.1, $\mathrm{p}=0.004$ ) and gross total resection (OR: $9.61,95 \% \mathrm{Cl}: 3.61-15.6, \mathrm{p}=0.002$ ), while NTR had better facial nerve outcome than GTR (OR: 1.5, 95\%Cl: 1.14-4.6, $p<0.0001$ ).

\section{Use of the Grading System to compare surgical approaches:}

We used the Vestibular Schwannoma Surgical Outcome Grading Scale to perform a non-weighted pooling of cases across those 40 of the 56 studies that included only one surgical approach or the other and used a simple statistical test of the difference between proportions. Overall, TL resulted in good HB function in $64.4 \%$ and RS in $57.1 \%$ ( $Z=4.12, p \leq .0001)$. GTR was performed slightly more often by surgeons using RS than TL (91.9\% vs $89.6 \% ; Z=2.23, p \leq .026)$. Table 3 presents the results using the grading system that combines EOR and HB facial nerve grade. For all six Grade outcomes, there was a significant difference between TL and RS. TL cases showed a higher rate of Grade A and Grade B results, the two best outcomes, while RS had a higher rate of Grade C outcomes as well as Grade D outcomes. Although percent of cases with Grades E or F were quite small, RS showed slightly lower rates for these two poorest results categories. That is, when a combination of extent of resection and facial nerve function is considered, TL outperformed RS in providing a good facial nerve function outcome with the greatest extent of resection. A further look showed that when GTR was performed, TL had a rate of good HB of $62.2 \%$ compared to RS with a rate of $53.7 \%(Z=4.52, p \leq .0001)$. Although GTR was reported more 
often when using RS than TL, good facial nerve results with GTR were more likely with the TL surgical approach. When STR was performed, RS outperformed for good HB result ( $94.5 \%$ vs $74.5 \%$, respectively; $Z=4.11, p \leq .0001)$. The difference for NTR was not significant. 
Table 1

Inclusion and Exclusion criteria's for our study

\begin{tabular}{|c|c|c|}
\hline $\begin{array}{l}\text { Study } \\
\text { Component }\end{array}$ & Inclusion & Exclusion \\
\hline \multirow[t]{3}{*}{ Participants } & - Adult patients ( $18-80$ years old $)$ & - History of previous Tumor resection \\
\hline & - Patients with Vestibular Schwannoma & - Infection \\
\hline & - Tumor size $\geq 2.5 \mathrm{~cm}$ & - Trauma/Fracture \\
\hline Intervention & $\begin{array}{l}\text { All Vestibular Schwannoma surgeries with } \\
\text { good clinical outcome }\end{array}$ & $\begin{array}{l}\text { - Stereotactic radiosurgery and/or } \\
\text { radiation therapy alone }\end{array}$ \\
\hline Comparators & $\begin{array}{l}\text { All Vestibular Schwannoma surgeries with } \\
\text { poor clinical outcome }\end{array}$ & $\begin{array}{l}\text { Studies that do not include any } \\
\text { patients in whom surgical } \\
\text { approaches were not mentioned }\end{array}$ \\
\hline \multirow[t]{2}{*}{ Outcomes } & $\begin{array}{l}\text {-Facial Nerve Outcome at } 1 \text {-year according } \\
\text { to House-Brackmann Scale }\end{array}$ & \multirow[t]{2}{*}{$\begin{array}{l}\text { Studies that did not facial nerve and } \\
\text { extent of resection as a potential } \\
\text { outcome }\end{array}$} \\
\hline & $\begin{array}{l}\text {-Extent of resection (Gross Total Resection, } \\
\text { Subtotal Resection, and Near Total } \\
\text { Resection) }\end{array}$ & \\
\hline \multirow{3}{*}{$\begin{array}{l}\text { Study } \\
\text { Design }\end{array}$} & \multirow{3}{*}{$\begin{array}{l}\text { RCTs, cohort studies, prospective or } \\
\text { retrospective studies with } \geq 10 \text { patients per } \\
\text { group }\end{array}$} & - In vitro studies, animal studies \\
\hline & & - Case reports \\
\hline & & $\begin{array}{l}\text { - Comparative studies with }<10 \\
\text { patients per treatment group }\end{array}$ \\
\hline \multirow[t]{8}{*}{ Publication } & \multirow{2}{*}{$\begin{array}{l}\text { Studies published in English in peer } \\
\text { reviewed journals, publicly published HTAs } \\
\text { or available FDA reports. }\end{array}$} & \multirow{3}{*}{$\begin{array}{l}\text { - Abstracts, editorials, letters } \\
\text { - Duplicate publications of the same } \\
\text { study which do not report on different } \\
\text { outcomes }\end{array}$} \\
\hline & & \\
\hline & \multirow{2}{*}{$\begin{array}{l}\text { (Each publication with a full review should } \\
\text { be indicated whether or not it is an industry } \\
\text { supported study.) }\end{array}$} & \\
\hline & & - Single reports from multicentre trials \\
\hline & \multirow[t]{4}{*}{$\begin{array}{l}\text { Databases to be searched: PubMed, } \\
\text { EMBASE, Cochrane, clinical trial.gov }\end{array}$} & $\begin{array}{l}\text { - Studies reporting on the technical } \\
\text { aspects of biologics use in fusion } \\
\text { surgery }\end{array}$ \\
\hline & & -White papers \\
\hline & & - Narrative and systematic reviews \\
\hline & & $\begin{array}{l}\text { - Articles identified as preliminary } \\
\text { reports when results are published in } \\
\text { later versions }\end{array}$ \\
\hline
\end{tabular}

Table 2: Baseline Characteristics of all included studies 


\begin{tabular}{|c|c|c|c|c|c|}
\hline \multirow[t]{2}{*}{ Author's Name and Year } & \multirow[t]{2}{*}{$\begin{array}{l}\text { No. of } \\
\text { Pts }\end{array}$} & \multirow[t]{2}{*}{$\begin{array}{l}\text { Surgical } \\
\text { Approach }\end{array}$} & \multirow[t]{2}{*}{$\begin{array}{l}\text { Degree of } \\
\text { Resection }\end{array}$} & \multicolumn{2}{|c|}{$\begin{array}{l}\text { House-Brackman } \\
\text { Scale } \\
\text { Good } \\
\text { Poor }\end{array}$} \\
\hline & & & & HII & III-VI \\
\hline \multirow{3}{*}{$\begin{array}{l}\text { Kirkpatrick et al } 1993 \\
\text { [20] }\end{array}$} & \multirow[t]{3}{*}{16} & TL: NA & GTR: 16 & \multirow[t]{3}{*}{ GTR: 4} & \multirow[t]{3}{*}{ GTR: 12} \\
\hline & & RS: NA & STR: 0 & & \\
\hline & & EX: NA & NTR: 0 & & \\
\hline \multirow[t]{3}{*}{ Wu et al. 2000 [75] } & \multirow[t]{3}{*}{40} & TL: 40 & \multirow[t]{3}{*}{ GTR: 39} & \multirow[t]{3}{*}{ GTR: 26} & \multirow[t]{3}{*}{ GTR: 13} \\
\hline & & RS: 0 & & & \\
\hline & & RL: 0 & & & \\
\hline \multirow[t]{3}{*}{ Jung et al 2000 [22] } & \multirow[t]{3}{*}{30} & TL: 0 & \multirow[t]{3}{*}{ GTR: 22} & \multirow[t]{3}{*}{ GTR: 8} & \multirow[t]{3}{*}{ GTR: 14} \\
\hline & & RS: 30 & & & \\
\hline & & RL: 0 & & & \\
\hline \multirow[t]{3}{*}{ Sluyter et al 2001 [23] } & \multirow[t]{3}{*}{99} & TL: 99 & \multirow[t]{3}{*}{ GTR: 91} & \multirow[t]{3}{*}{ GTR: 46} & \multirow[t]{3}{*}{ GTR: 45} \\
\hline & & RS: 0 & & & \\
\hline & & RL: 0 & & & \\
\hline \multirow{3}{*}{$\begin{array}{l}\text { Mamikoglu et al } 2002 \\
\text { [24] }\end{array}$} & \multirow[t]{3}{*}{81} & TL: 81 & \multirow[t]{3}{*}{ GTR: 77} & \multirow[t]{3}{*}{ GTR: 35} & \multirow[t]{3}{*}{ GTR: 42} \\
\hline & & RS: 0 & & & \\
\hline & & RL: 0 & & & \\
\hline \multirow[t]{3}{*}{ Lee et al 2002 [25] } & \multirow[t]{3}{*}{36} & TL: 0 & \multirow[t]{3}{*}{ GTR: 11} & GTR: 7 & GTR: 4 \\
\hline & & RS: 36 & & & \\
\hline & & RL: 0 & & & \\
\hline Iwai et al 2003 [26] & 14 & TL: 1 & STR: 14 & STR: 10 & STR: 4 \\
\hline & & RS: 13 & & & \\
\hline & & EX: 0 & & & \\
\hline Yamakami et al 2004 & 50 & TL: 0 & GTR: 43 & GTR: 36 & GTR: 7 \\
\hline & & RS: 50 & & & \\
\hline & & RL: 0 & & & \\
\hline Roland JT et al 2004 [28] & 56 & TL: 46 & GTR: 35 & GTR: 34 & GTR: 4 \\
\hline & & RS: 10 & STR: 6 & STR: 6 & STR: 0 \\
\hline
\end{tabular}




\begin{tabular}{|c|c|c|c|c|c|}
\hline & & EX: 0 & NTR: 5 & NTR: 2 & NTR: 3 \\
\hline \multirow[t]{3}{*}{ Sanna et al 2004 [29] } & 175 & TL: 175 & GTR: 150 & GTR: 52 & GTR: 98 \\
\hline & & RS: 0 & & & \\
\hline & & $\mathrm{RL}: 0$ & & & \\
\hline \multirow[t]{3}{*}{ Gerganov et al 2005 [30] } & 18 & TL: 0 & GTR: 11 & GTR: 4 & GTR: 7 \\
\hline & & RS: 18 & & & \\
\hline & & $\mathrm{RL}: 0$ & & & \\
\hline \multirow[t]{3}{*}{ Darwish et al 2005 [31] } & 35 & TL: 0 & GTR: 35 & GTR: 7 & GTR: 28 \\
\hline & & RS: 35 & & & \\
\hline & & $\mathrm{RL}: 0$ & & & \\
\hline \multirow{3}{*}{$\begin{array}{l}\text { Raftopoulos et al } 2005 \\
\text { [32] }\end{array}$} & 16 & TL: 1 & GTR: 11 & GTR: 11 & GTR: 0 \\
\hline & & RS: 15 & & & \\
\hline & & RL: 0 & & & \\
\hline \multirow[t]{3}{*}{ Anderson et al 2005 [33] } & 71 & TL: 50 & GTR: 68 & GTR: 50 & GTR: 18 \\
\hline & & RS: 21 & & & \\
\hline & & $\mathrm{RL}: 0$ & & & \\
\hline \multirow[t]{3}{*}{ Zhang X et al 2005 [16] } & 105 & TL: 0 & GTR: 91 & GTR: 51 & GTR: 40 \\
\hline & & RS: 105 & & & \\
\hline & & $\mathrm{RL}: 0$ & & & \\
\hline \multirow[t]{3}{*}{ Jain et al 2005 [34] } & 145 & TL: 0 & GTR: 142 & GTR: 43 & GTR: 99 \\
\hline & & RS: 145 & & & \\
\hline & & $\mathrm{RL}: 0$ & & & \\
\hline \multirow[t]{3}{*}{ Samii et al 2006 [35] } & 92 & TL: 0 & GTR: 88 & GTR: 46 & GTR: 42 \\
\hline & & RS: 92 & & & \\
\hline & & $\mathrm{RL}: 0$ & & & \\
\hline \multirow[t]{3}{*}{ Park et al 2006 [36] } & 50 & TL: 6 & GTR: 9 & GTR: 5 & GTR: 4 \\
\hline & & RS: 43 & STR: 31 & STR: 27 & STR: 4 \\
\hline & & $\mathrm{RL}: 1$ & NTR: 8 & NTR: 5 & NTR: 3 \\
\hline \multirow[t]{2}{*}{ Liu et al 2007 [37] } & 19 & TL: 0 & GTR: 12 & GTR: 8 & GTR: 4 \\
\hline & & RS: 19 & & & \\
\hline
\end{tabular}




\begin{tabular}{|c|c|c|c|c|c|}
\hline & & $\mathrm{RL}: 0$ & & & \\
\hline Yang et al 2008 [38] & 110 & TL: 0 & STR: 61 & STR: 58 & STR: 3 \\
\hline & & RS: 110 & & & \\
\hline & & RL: 0 & & & \\
\hline Fuentes et al 2008 [39] & 8 & TL: 0 & STR: 8 & STR: 7 & STR: 1 \\
\hline & & RS: 8 & & & \\
\hline & & RL: 0 & & & \\
\hline Strauss et al 2008 [40] & 10 & TL: 0 & GTR: 7 & GTR: 4 & GTR: 3 \\
\hline & & RS: 10 & & & \\
\hline & & $\mathrm{RL}: 0$ & & & \\
\hline Chen et al 2009 [8] & 39 & TL: 0 & NR & GTR: 27 & GTR: 12 \\
\hline & & RS: 39 & & & \\
\hline & & RL: 0 & & & \\
\hline Godefroy WP et al 2009 & 50 & TL: 50 & GTR: 13 & GTR: 9 & GTR: 4 \\
\hline & & RS: 0 & STR: 8 & STR: 8 & STR: 0 \\
\hline & & EX: 0 & NTR: 29 & NTR:22 & NTR: 7 \\
\hline Samii M et al 2010 [42] & 36 & TL: 0 & GTR: 36 & GTR: 16 & GTR: 20 \\
\hline & & RS: 36 & STR: 0 & & \\
\hline & & EX: 0 & NTR: 0 & & \\
\hline Zhao et al 2010 [43] & 89 & TL: 0 & GTR: 38 & GTR: 20 & GTR: 18 \\
\hline & & RS: 89 & & & \\
\hline & & $\mathrm{RL}: 0$ & & & \\
\hline Talfer et al 2010 [44] & 51 & TL: 51 & GTR: NR & GTR: 25 & GTR: 26 \\
\hline & & RS: 0 & & & \\
\hline & & RL: 0 & & & \\
\hline Bloch et al 2011 [45] & 100 & TL: NR & GTR: NR & GTR: 44 & GTR: 56 \\
\hline & & RS: NR & & & \\
\hline & & RL: NR & & & \\
\hline Di Maio et al 2011 [46] & 47 & TL: 0 & GTR: 41 & GTR: 38 & GTR: 3 \\
\hline & & RS: 47 & & & \\
\hline
\end{tabular}




\begin{tabular}{|c|c|c|c|c|c|}
\hline \multirow{2}{*}{ Haque et al 2011 [47] } & \multirow[b]{2}{*}{151} & \multicolumn{2}{|l|}{$\mathrm{RL}: 0$} & \multirow[b]{2}{*}{ GTR: 53} & \multirow[b]{2}{*}{ GTR: 2} \\
\hline & & TL: NR & GTR: 55 & & \\
\hline & & RS: NR & STR: 96 & STR: 93 & STR: 3 \\
\hline & & RL: NR & NTR: 0 & NTR: 0 & NTR: 0 \\
\hline \multirow{3}{*}{$\begin{array}{l}\text { Langenberg RV et al } \\
2011 \text { [48] }\end{array}$} & \multirow[t]{3}{*}{42} & TL: NR & GTR: 0 & \multirow[t]{3}{*}{ STR: 34} & \multirow[t]{3}{*}{ STR: 16} \\
\hline & & RS: NR & STR: 42 & & \\
\hline & & EX: 0 & NTR: 0 & & \\
\hline \multirow[t]{3}{*}{ Nuseir A et al 2011 [49] } & \multirow[t]{3}{*}{232} & TL: 226 & GTR: 163 & \multirow[t]{3}{*}{ STR: 40} & \multirow[t]{3}{*}{ STR: 15} \\
\hline & & RS: 2 & STR: 69 & & \\
\hline & & EX: 4 & NTR: 0 & & \\
\hline \multirow[t]{3}{*}{ Silva J et al 2012 [50] } & \multirow[t]{3}{*}{29} & TL: 0 & GTR: 29 & \multirow[t]{3}{*}{ GTR: 13} & \multirow[t]{3}{*}{ GTR: 16} \\
\hline & & RS: 29 & STR: 0 & & \\
\hline & & $\mathrm{RL}: 0$ & NTR: 0 & & \\
\hline \multirow[t]{3}{*}{ Pan et al 2012 [51] } & \multirow[t]{3}{*}{35} & TL: NR & GTR: NR & \multirow[t]{3}{*}{ STR: 16} & \multirow[t]{3}{*}{ STR: 2} \\
\hline & & RS: NR & STR: 18 & & \\
\hline & & RL: NR & NTR: NR & & \\
\hline \multirow[t]{3}{*}{ Zhang Zh. et al 2012 [52] } & \multirow[t]{3}{*}{115} & TL: 115 & GTR: 103 & \multirow[t]{3}{*}{ GTR: 37} & \multirow[t]{3}{*}{ GTR: 66} \\
\hline & & RS: 0 & & & \\
\hline & & RL: 0 & & & \\
\hline \multirow{3}{*}{$\begin{array}{l}\text { Schwartz M et al } 2013 \\
\text { [53] }\end{array}$} & \multirow[t]{3}{*}{400} & TL: 400 & GTR: 325 & GTR: 250 & GTR: 75 \\
\hline & & RS: 0 & STR: 31 & STR: 30 & STR: 1 \\
\hline & & RL: 0 & NTR: 44 & NTR: 43 & NTR: 1 \\
\hline \multirow[t]{3}{*}{ Nonaka et al 2013 [54] } & \multirow[t]{3}{*}{62} & TL: NR & GTR: 28 & \multirow[t]{3}{*}{ GTR: 19} & \multirow[t]{3}{*}{ GTR: 9} \\
\hline & & RS: NR & & & \\
\hline & & RL: NR & & & \\
\hline Porter et al 2013 [55] & 153 & TL: 153 & GTR: 55 & GTR: 43 & GTR: 12 \\
\hline & & RS: 0 & & & \\
\hline & & RL: 0 & & & \\
\hline Lim et al 2013 [56] & 27 & TL: 0 & GTR: NR & GTR: 20 & GTR: 7 \\
\hline & & RS: 27 & & & \\
\hline
\end{tabular}




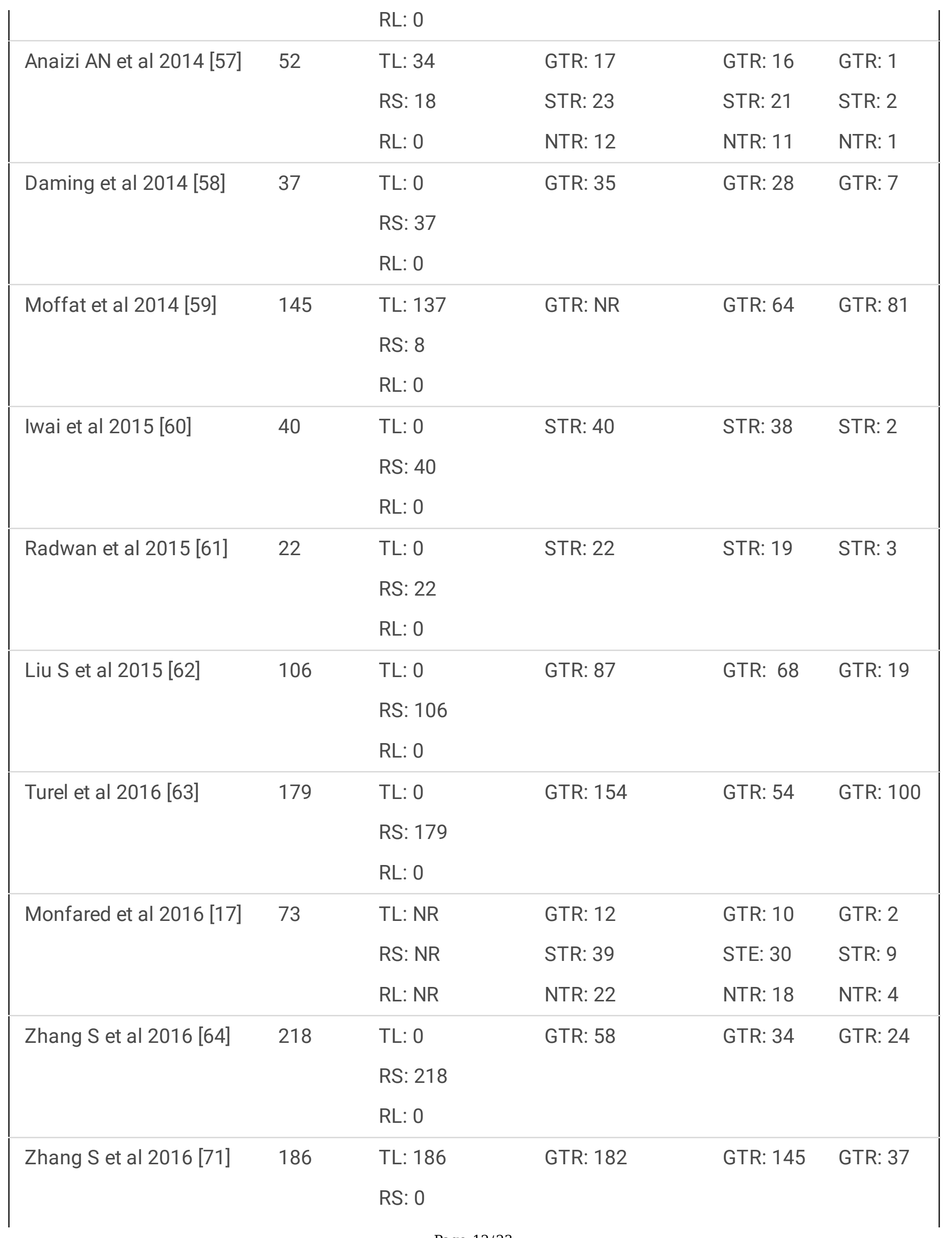




\begin{tabular}{|c|c|c|c|c|c|}
\hline & & $\mathrm{RL}: 0$ & & & \\
\hline Daniel et al 2017 [15] & 32 & TL: 0 & STR: 32 & STR: 32 & STR: 0 \\
\hline & & RS: 32 & & & \\
\hline & & $\mathrm{RL}: 0$ & & & \\
\hline Huang et al 2017 [65] & 657 & TL: 0 & GTR: 556 & GTR: 183 & GTR: 373 \\
\hline & & RS: 657 & & & \\
\hline & & RL: 0 & & & \\
\hline Boublata et al 2017 [66] & 151 & TL: 0 & GTR: 126 & GTR: 103 & GTR: 23 \\
\hline & & RS: 151 & & & \\
\hline & & $\mathrm{RL}: 0$ & & & \\
\hline Hoshide et al 2018 [67] & 45 & TL: 0 & GTR: 29 & GTR: 25 & GTR: 4 \\
\hline & & RS: 45 & & & \\
\hline & & RL: 0 & & & \\
\hline Breun et al 2019 [68] & 320 & TL: 0 & GTR: 196 & GTR: 187 & GTR: 9 \\
\hline & & RS: 320 & & & \\
\hline & & RL: 0 & & & \\
\hline Grinblat G et al 2020 [69] & 389 & TL: 374 & GTR: 301 & GTR: 102 & GTR: 199 \\
\hline & & RS: 0 & STR: 51 & & \\
\hline & & ET: 15 & NTR: 37 & & \\
\hline Schneider JR et al 2020 & 37 & TL: 0 & GTR: 27 & GTR: 21 & GTR: 6 \\
\hline & & RS: 37 & STR: 0 & STR: 0 & STR: 0 \\
\hline & & RL: 0 & NTR: 10 & NTR: 10 & NTR: 0 \\
\hline
\end{tabular}

TL: Trans-labyrinthine, EX: Extended trans-labyrinthine, RS: Retro sigmoid, GTR: Gross Total Resection, NTR: Near Total Resection, STR: Sub-Total Resection

Table 3. Comparison of translabyrinthine (TL) and retrosigmoid (RS) surgical approaches using new grading system based on 40 studies in which only TL or only RS were used. 


\begin{tabular}{|llll|}
\hline Acoustic Neuroma Grading System Outcome & $\begin{array}{l}\text { TL } \\
\text { N=1198 }\end{array}$ & $\begin{array}{l}\text { RS } \\
\text { N=2127 }\end{array}$ & Statistical Significance* \\
\hline Class A (GTR+Good HB) & $55.8 \%$ & $49.4 \%$ & $Z=3.54, p \leq .0004$ \\
\hline Class B (NTR+Good HB) & $5.4 \%$ & $0.5 \%$ & $Z=9.11, p \leq .0001$ \\
\hline Class C (STR+Good HB) & $3.2 \%$ & $7.2 \%$ & $Z=4.75, p \leq .0001$ \\
\hline Class D (GTR+Poor HB) & $33.9 \%$ & $42.5 \%$ & $Z=4.87, p \leq .0001$ \\
\hline Class E (NTR+Poor HB) & $0.7 \%$ & $0.0 \%$ & $Z=3.75, p \leq .0002$ \\
\hline Class F (STR+Poor HB) & $1.1 \%$ & $0.4 \%$ & $Z=2.41, p \leq .016$ \\
\hline *Z-test for the difference between two proportions & & \\
\hline GTR=Gross total resection, NTR=Near total resection, STR=Subtotal resection \\
\hline Good HB=House-Brackmann facial nerve grade I or II, Poor HB=HB grades III-VI \\
\hline
\end{tabular}

\section{Publication Bias:}

We found some evidence of publication bias, as suggested by slight asymmetry of the funnel plot

(Egger test, $\mathrm{z}=-2.48 ; \mathrm{p}=0.009$ ) and association between effect sizes and corresponding sampling variances (Begg test, $z=-1.49 ; p=0.04$ ). According to the trim-and-fill method to correct for publication bias, the association in terms of clinical outcome was still significant after imputing 2 possible missing studies (adjusted OR: 6.18; 95\% Cl, 3.46-11.9; $\mathrm{p}<0.0001$ ), suggesting no potential role for small-study effects or publication bias on the meta-analysis results.

\section{Discussion}

The ideal treatment for large VS is complete surgical removal with preservation of facial nerve function. However, these large tumors pose challenges and increase postoperative complication rates compared to smaller VS because patients may present with intracranial hypertension, multiple cranial nerve deficits, and/or signs of brainstem/cerebellum compression. Therefore, the choice of EOR for large VS remains unsettled. Most published studies use facial nerve function alone as the measure of outcome.[33, 41, 45, 53] However, the current study presents and shows the usefulness of a novel surgical outcome scoring system incorporating both EOR and facial nerve function for evaluating the functional outcome in VS surgery.

STR is usually adopted as a nerve-centered approach, hence mostly performed in patients having large tumors adherent to the facial nerve.[72] However, patients with STR have a higher likelihood of tumor regrowth compared to NTR or GTR.[73, 74] GTR is considered to be optimal to prevent tumor recurrence, while NTR also decreases recurrence rate due to the fact that it reduces blood supply or removes critical tumor mass to prevent regrowth. Therefore, the Vestibular Schwannoma Surgical Outcome Grading Scale 
presented here takes this into account in defining outcome and may provide a standard method of evaluating surgical outcome among patients with VS worldwide. Hence, Grade A represents the ideal outcome and Grade $F$ the worst outcome obtained from VS surgery for large to giant tumors.

Previous studies have shown that good facial nerve results can be obtained with GTR and NTR. For example, Strickland et al ${ }^{74}$ reported that patients with NTR achieved better tumor control and higher likelihood of facial nerve recovery compared with STR, while Samii et al [42] reported excellent facial nerve outcome in $75 \%$ of patients following GTR of large VS. Our results are consistent with these and other prior reports in that STR is associated with better facial nerve outcomes when looked at in isolation. If we consider, EOR and HB facial nerve grade as independent variables, patients undergoing STR had a higher rate of good facial nerve outcome (61.0\%) (Grade C) than NTR (59.9\%) (Grade B) and GTR (52.2\%) (Grade A). This concurs with a previously conducted meta-analysis by Gurgel et al [11] which found that STR was better than NTR and NTR was significantly better than GTR with regard to facial nerve outcomes. These results, as well as those in the current study, may be confounded by pre-operative HB grade, tumor size, cranial nerve deficits, adherence to facial nerve and other factors for which data were not available by individual patient for the meta-analysis. However, use of a grading system that combines HB grade with EOR can provide a different perspective on overall outcome.

There also remains a question about whether choice of surgical approach makes a difference for optimal facial nerve outcome [11]. The current meta-analysis suggested that the two most common approaches, translabyrinthine and retrosigmoid, were not significantly different when assessing facial nerve outcome or EOR alone. However, a difference could be shown using the new grading system. A further nonweighted pooling of cases across those 40 studies that included only one surgical approach or the other and use of a simple statistical test of the difference between proportions also showed the usefulness of the grading system, with TL outperforming RS in obtaining the two best outcome grades. Results might also be interpreted to suggest that if the surgeon is planning to do a subtotal resection, which is inherently less likely to damage the facial nerve, RS should have a higher proportion of good facial nerve outcome, but if planning to attempt to remove the entire tumor TL will yield better facial nerve results. That is, surgeon intention regarding EOR is a confounder for facial nerve outcome as those not intending on trying to remove the whole tumor will generally be less inclined to go through the longer and more demanding translab approach. A significant caveat here is surgeon/inherent bias as these 40 studies included only surgeries performed by surgeons who preferred and used one surgical technique over the other, so that the differences between surgical approaches is confounded by possible differences between surgeons, their experience, and even possibly their patient populations.

A meta-analysis pools data from different studies and because of that there are a number of potential limitations: (i) inherent bias of the surgeons regarding EOR as well as surgical approach, (ii) variability in tumor size, (iii) degree of resection, (iv) length of follow-up, (v) varying surgical protocols among surgeons worldwide, and (vi) heterogenous populations. In addition, as noted above, it may not be possible in a meta-analysis to account for confounding factors such as pre-operative facial nerve grade, cranial nerve deficits, adherence of tumor to facial nerve or other individual patient risk factors. However, 
these are issues that will likely always remain problematic in trying to compare results across different studies of very large VS. Our proposed Acoustic Tumor Grading System would lessen some of the variability in reporting results while taking into account the two most important measures of success.

\section{Conclusion}

Complete surgical resection with preservation of facial nerve is the gold standard for large VS. However, a nerve-centered approach using near total and subtotal extent of resection is gaining in prominence. This new proposed grading system may help reduce inconsistencies in reporting of outcomes and help when strategizing management of such patients.

\section{Abbreviations}

VS

Vestibular Schwannoma

STR

Subtotal resection

NTR

Near Total Resection

GTR

Gross Total Resection

TL

translabyrinthine

RS

RetroSigmoid

$\mathrm{RL}$

Retrolabyrinthine

EX

Extended translabyrinthine.

\section{Declarations}

Submission Statement: This manuscript is original and has not been submitted elsewhere in part or in whole

Running Title: Vestibular Schwannoma Surgical Outcome Grading Scale

Declarations:

Acknowledgments: None

Funding: None. 
Conflict of interest/Competing Interest: None

Ethics Approval: Not required

Availability of data and material: All data generated or analyzed during this study are included in

this published article

Consent to Participate: Not Applicable

Consent to Publish: Not Applicable

Authors Contribution: Nida Fatima, Gregory P Lekovic: Manuscript Writing; Gregory P Lekovic, Nida Fatima: Data Collection, Nida Fatima and Gregory P Lekovic: Data/Statistical Analysis; Nida Fatima, Gregory P Lekovic: Editing and Final Revision; Supervision: Gregory P Lekovic

Previous Presentation: None

\section{References}

1. Luetje CM, Whittaker CK, Callaway LA, Veraga G, HISTOLOGICAL ACOUSTIC TUMOR INVOLVEMENT OF THE VIIth NERVE AND MULTICENTRIC ORIGIN IN THE VIIIth NERVE (1983) : The Laryngoscope 93:1133???1139. https://doi.org/10.1288/00005537-198309000-00004

2. Stangerup S-E, Caye-Thomasen P (2012) Epidemiology and Natural History of Vestibular Schwannomas. Otolaryngol Clin North Am 45:257-268. https://doi.org/10.1016/j.otc.2011.12.008

3. Khrais T, Romano G, Sanna M (2008) Nerve origin of vestibular schwannoma: a prospective study. J Laryngol Otol 122:128-131. https://doi.org/10.1017/S0022215107001028

4. Tripathee S, Zhang J, Xiong M (2016) Risk factors of microtia: A systematic review and metaanalysis. Eur J Plast Surg 39:335-344. https://doi.org/10.1007/s00238-016-1196-4

5. Elsmore AJ, Mendoza ND (2002) The operative learning curve for vestibular schwannoma excision via the retrosigmoid approach. Br J Neurosurg 16:448-455. https://doi.org/10.1080/0268869021000030249

6. Samii M, Matthies C (1997) Management of 1000 Vestibular Schwannomas (Acoustic Neuromas): The Facial Nerve-Preservation and Restitution of Function. Neurosurgery 40:684-695. https://doi.org/10.1097/00006123-199704000-00006

7. Misra B, Purandare H, Ved R et al (2009) Current treatment strategy in the management of vestibular schwannoma. Neurol India 57:257. https://doi.org/10.4103/0028-3886.53263

8. Cheng S, Naidoo Y, da Cruz M, Dexter M (2009) Quality of life in postoperative vestibular schwannoma patients. Laryngoscope 119:2252-2257. https://doi.org/10.1002/lary.20217

9. Arriaga MA, Chen DA (2001) Facial Function in Hearing Preservation Acoustic Neuroma Surgery. Arch Otolaryngol Head Neck Surg 127:543. https://doi.org/10.1001/archotol.127.5.543 
10. Arts HA, Telian SA, El-Kashlan H, Thompson BG (2006) Hearing Preservation and Facial Nerve Outcomes in Vestibular Schwannoma Surgery: Results Using the Middle. Cranial Fossa Approach: Otology \& Neurotology 27:234-241. https://doi.org/10.1097/01.mao.0000185153.54457.16

11. Gurgel RK, Dogru S, Amdur RL, Monfared A (2012) Facial nerve outcomes after surgery for large vestibular schwannomas: do surgical approach and extent of resection matter? FOC 33:E16. https://doi.org/10.3171/2012.7.FOCUS12199

12. Dandy WE, RESULTS OF REMOVAL OF ACOUSTIC TUMORS BY THE UNILATERAL APPROACH (1941) Arch Surg 42:1026. https://doi.org/10.1001/archsurg.1941.01210120061006

13. Cushing $H$ (1932) Intracranial Tumor. In: Springfield

14. House WF (1968) Partial tumor removal and recurrence in acoustic tumor surgery. Arch Otolaryngol 88:644-654. https://doi.org/10.1001/archotol.1968.00770010646013

15. Daniel RT, Tuleasca C, George M et al (2017) Preserving normal facial nerve function and improving hearing outcome in large vestibular schwannomas with a combined approach: planned subtotal resection followed by gamma knife radiosurgery. Acta Neurochir 159:1197-1211. https://doi.org/10.1007/s00701-017-3194-0

16. Zhang X, Fei Z, Chen Y-J et al (2005) Facial nerve function after excision of large acoustic neuromas via the suboccipital retrosigmoid approach. Journal of Clinical Neuroscience 12:405-408. https://doi.org/10.1016/j.jocn.2004.03.042

17. Monfared A, Corrales CE, Theodosopoulos PV et al (2016) Facial Nerve Outcome and Tumor Control Rate as a Function of Degree of Resection in Treatment of Large Acoustic Neuromas. Neurosurgery 79:194-203. https://doi.org/10.1227/NEU.0000000000001162

18. Nakatomi H, Jacob JT, Carlson ML et al (2020) Long-term risk of recurrence and regrowth after gross-total and subtotal resection of sporadic vestibular schwannoma. J Neurosurg 133:1052-1058. https://doi.org/10.3171/2016.11.JNS16498

19. Moher D, Liberati A, Tetzlaff J, Altman DG (2009) Preferred Reporting Items for Systematic Reviews and Meta-Analyses: The PRISMA Statement. J Clin Epidemiol 62:1006-1012. https://doi.org/10.1016/j.jclinepi.2009.06.005

20. Kirkpatrick PJ, Tierney P, Gleeson MJ, Strong AJ (1993) Acoustic tumour volume and the prediction of facial nerve functional outcome from intraoperative monitoring. $\mathrm{Br} \mathrm{J}$ Neurosurg 7:657-664. https://doi.org/10.3109/02688699308995095

21. Wu ZB, Yu CJ, Guan SS (2005) Posterior petrous meningiomas: 82 cases. J Neurosurg 102:284-289. https://doi.org/10.3171/jns.2005.102.2.0284

22. Jung S, Kang S-S, Kim T-S et al (2000) Current surgical results of retrosigmoid approach in extralarge vestibular schwannomas. Surg Neurol 53:370-378. https://doi.org/10.1016/S0090-3019(00)001968

23. Sluyter S, Graamans K, Tulleken CAF, Van Veelen CWM (2001) Analysis of the results obtained in 120 patients with large acoustic neuromas surgically treated via the translabyrinthine-transtentorial approach. J Neurosurg 94:61-66. https://doi.org/10.3171/jns.2001.94.1.0061 
24. Mamikoglu B, Wiet RJ, Esquivel CR (2002) Translabyrinthine Approach for the Management of Large and Giant Vestibular Schwannomas. Otology \& Neurotology 23:224-227. https://doi.org/10.1097/00129492-200203000-00020

25. Lee SH, Willcox TO Jr, Buchheit WA (2002) Current Results of the Surgical Management of Acoustic Neuroma. Skull Base 12:189-196. https://doi.org/10.1055/s-2002-35750-1

26. Iwai $Y$, Yamanaka $K$, Ishiguro $T$ (2003) Surgery combined with radiosurgery of large acoustic neuromas. Surg Neurol 59:283-289. https://doi.org/10.1016/S0090-3019(03)00025-9

27. Yamakami I (2004) Removal of large acoustic neurinomas (vestibular schwannomas) by the retrosigmoid approach with no mortality and minimal morbidity. Journal of Neurology, Neurosurgery \& Psychiatry 75:453-458. https://doi.org/10.1136/jnnp.2003.010827

28. Roland JT, Fishman AJ, Golfinos JG et al (2004) Cranial Nerve Preservation in Surgery for Large Acoustic Neuromas. Skull Base 14:85-90. https://doi.org/10.1055/s-2004-828699

29. Sanna M, Russo A, Falcioni M et al (2004) Enlarged Translabyrinthine Approach for the Management of Large and Giant Acoustic Neuromas: A Report of 175 Consecutive Cases. Ann Otol Rhinol Laryngol 113:319-328. https://doi.org/10.1177/000348940411300412

30. Gerganov VM, Romansky KV, Bussarsky VA et al (2005) Endoscope-Assisted Microsurgery of Large Vestibular Schwannomas. Minim Invasive Neurosurg 48:39-43. https://doi.org/10.1055/s-2004830171

31. Darwish BS, Bird PA, Goodisson DW et aIORIGINALARTICLE FACIAL NERVE FUNCTION AND HEARING PRESERVATION AFTER. 4

32. Raftopoulos C, Abu Serieh B, Duprez T et al (2005) Microsurgical results with large vestibular schwannomas with preservation of facial and cochlear nerve function as the primary aim. Acta Neurochir (Wien) 147:697-706. https:// doi.org/10.1007/s00701-005-0544-0

33. Anderson DE, Leonetti J, Wind JJ et al (2005) Resection of large vestibular schwannomas: facial nerve preservation in the context of surgical approach and patient-assessed outcome. J Neurosurg 102:643-649. https://doi.org/10.3171/jns.2005.102.4.0643

34. Jain V, Mehrotra N, Sahu R et al (2005) Surgery of vestibular schwannomas: An institutional experience. Neurol India 53:41. https://doi.org/10.4103/0028-3886.15052

35. Samii M, Gerganov V, Samii A (2006) Improved preservation of hearing and facial nerve function in vestibular schwannoma surgery via the retrosigmoid approach in a series of 200 patients. JNS 105:527-535. https://doi.org/10.3171/jns.2006.105.4.527

36. Park C-K, Kim D-C, Park S-H et al (2006) Microhemorrhage, a possible mechanism for cyst formation in vestibular schwannomas. JNS 105:576-580. https://doi.org/10.3171/jns.2006.105.4.576

37. Liu B, Tian Y, Liu W et al (2007) Intraoperative facial motor evoked potentials monitoring with transcranial electrical stimulation for preservation of facial nerve function in patients with large acoustic neuroma. Chin Med J (Engl) 120:323-325

38. Yang I, Sughrue ME, Han SJ et al (2009) Facial nerve preservation after vestibular schwannoma Gamma Knife radiosurgery. J Neurooncol 93:41-48. https://doi.org/10.1007/s11060-009-9842-3 
39. Fuentes S, Arkha Y, Pech-Gourg G et al (2008) Management of Large Vestibular Schwannomas by Combined Surgical Resection and Gamma Knife Radiosurgery.. In: In: Régis J, Roche P-H (eds) Progress in Neurological Surgery. KARGER, Basel, pp 79-82

40. Strauss C, Bischoff B, Romstöck J et al (2008) Hearing preservation in medial vestibular schwannomas. JNS 109:70-76. https://doi.org/10.3171/JNS/2008/109/7/0070

41. Godefroy WP, Hoekstra ER, Malessy MJA (2009) Surgery for Large Vestibular Schwannoma: Residual Tumor and Outcome. 30:6

42. Samii M, Gerganov VM, Samii A (2010) Functional outcome after complete surgical removal of giant vestibular schwannomas: Clinical article. JNS 112:860-867.

https://doi.org/10.3171/2009.7.JNS0989

43. Zhao X, Wang Z, Ji Y et al (2010) Long-term facial nerve function evaluation following surgery for large acoustic neuromas via retrosigmoid transmeatal approach. Acta Neurochir 152:1647-1652. https://doi.org/10.1007/s00701-010-0705-7

44. Talfer S, Dutertre G, Conessa $C$ et al (2010) Surgical treatment of large vestibular schwannomas (stages III and IV). European Annals of Otorhinolaryngology, Head and Neck Diseases 127:63-69. https://doi.org/10.1016/j.anorl.2010.03.003

45. Bloch O, Sughrue ME, Kaur R et al (2011) Factors associated with preservation of facial nerve function after surgical resection of vestibular schwannoma. J Neurooncol 102:281-286. https://doi.org/10.1007/s11060-010-0315-5

46. Di Maio S, Malebranche AD, Westerberg B, Akagami R (2011) Hearing Preservation After Microsurgical Resection of Large Vestibular Schwannomas. Neurosurgery 68:632-640. https://doi.org/10.1227/NEU.0b013e31820777b1

47. Haque R, Wojtasiewicz TJ, Gigante PR et al (2011) Efficacy of facial nerve-sparing approach in patients with vestibular schwannomas: Clinical article. JNS 115:917-923. https://doi.org/10.3171/2011.7.JNS101921

48. van de Langenberg R, Hanssens PEJ, van Overbeeke JJ et al (2011) Management of large vestibular schwannoma. Part I. Planned subtotal resection followed by Gamma Knife surgery: radiological and clinical aspects: Clinical article. JNS 115:875-884. https://doi.org/10.3171/2011.6.JNS101958

49. Nuseir A, Sequino G, De Donato $G$ et al (2012) Surgical management of vestibular schwannoma in elderly patients. Eur Arch Otorhinolaryngol 269:17-23. https://doi.org/10.1007/s00405-011-1566-2

50. Silva J, Cerejo A, Duarte F et al (2012) Surgical Removal of Giant Acoustic Neuromas. World Neurosurgery 77:731-735. https://doi.org/10.1016/j.wneu.2011.08.019

51. Pan H-C, Sheehan J, Sheu M-L et al (2012) Intracapsular decompression or radical resection followed by Gamma Knife surgery for patients harboring a large vestibular schwannoma: Clinical article. JNS 117:69-77. https://doi.org/10.3171/2012.6.GKS12697

52. Zhang Z, Wang Z, Huang Q et al (2012) Removal of Large or Giant Sporadic Vestibular Schwannomas via Translabyrinthine Approach: A Report of 115 Cases. ORL 74:271-277. https://doi.org/10.1159/000343791 
53. Schwartz MS, Kari E, Strickland BM et al (2013) Evaluation of the Increased Use of Partial Resection of Large Vestibular Schwanommas: Facial Nerve Outcomes and Recurrence/Regrowth Rates. Otology \& Neurotology 34:1456-1464. https://doi.org/10.1097/MA0.0b013e3182976552

54. Nonaka Y, Fukushima T, Watanabe K et al (2013) Contemporary Surgical Management of Vestibular Schwannomas: Analysis of Complications and Lessons Learned Over the Past Decade. Operative Neurosurgery 72:ons103-ons115. https://doi.org/10.1227/NEU.0b013e3182752b05

55. Porter RG, LaRouere MJ, Kartush JM et al (2013) Improved Facial Nerve Outcomes Using an Evolving Treatment Method for Large Acoustic Neuromas. Otology \& Neurotology 34:304-310. https://doi.org/10.1097/MA0.0b013e31827d07d4

56. Lim SL, Hieng AWS (2013) Review of an 11-year Experience in Retrosigmoid Approach for Treatment of Acoustic Neuromas. 68:6

57. Anaizi AN, Gantwerker EA, Pensak ML, Theodosopoulos PV (2014) Facial Nerve Preservation Surgery for Koos Grade 3 and 4 Vestibular Schwannomas. Neurosurgery 75:671-677. https://doi.org/10.1227/NEU.0000000000000547

58. Daming C, Yiwen S, Bin Z et al (2014) Large Vestibular Schwannoma Resection Through the Suboccipital Retrosigmoid Keyhole Approach. Journal of Craniofacial Surgery 25:463-468. https://doi.org/10.1097/SCS.0000000000000528

59. Moffat DA, Parker RA, Hardy DG, Macfarlane R (2014) Factors affecting final facial nerve outcome following vestibular schwannoma surgery. J Laryngol Otol 128:406-415. https://doi.org/10.1017/S0022215114000541

60. Iwai Y, Ishibashi K, Watanabe $Y$ et al (2015) Functional Preservation After Planned Partial Resection Followed by Gamma Knife Radiosurgery for Large Vestibular Schwannomas. World Neurosurgery 84:292-300. https://doi.org/10.1016/j.wneu.2015.03.012

61. Radwan H, Eisenberg MB, Sandberg Knisely JP et al (2016) Outcomes in Patients with Vestibular Schwannoma after Subtotal Resection and Adjuvant Radiosurgery. Stereotact Funct Neurosurg 94:216-224. https://doi.org/10.1159/000447520

62. Liu S, Jiang $\mathrm{W}$, Zhang $\mathrm{H}$ et al (2015) Intraoperative neuromonitoring for removal of large vestibular schwannoma: Facial nerve outcome and predictive factors. Clin Neurol Neurosurg 133:83-89. https://doi.org/10.1016/j.clineuro.2015.03.016

63. Turel M, D'Souza W, Chacko A, Rajshekhar V (2016) Giant vestibular schwannomas: Surgical nuances influencing outcome in 179 patients. Neurol India 64:478. https://doi.org/10.4103/00283886.181558

64. Zhang S, Liu W, Hui X, You C (2016) Surgical Treatment of Giant Vestibular Schwannomas: Facial Nerve Outcome and Tumor Control. World Neurosurgery 94:137-144. https://doi.org/10.1016/j.wneu.2016.06.119

65. Huang MJ, Kano H, Mousavi SH et al (2017) Stereotactic radiosurgery for recurrent vestibular schwannoma after previous resection. J Neurosurg 126:1506-1513. https://doi.org/10.3171/2016.5.JNS1645 
66. Boublata L, Belahreche M, Ouchtati R et al (2017) Facial Nerve Function and Quality of Resection in Large and Giant Vestibular Schwannomas Surgery Operated By Retrosigmoid Transmeatal Approach in Semi-sitting Position with Intraoperative Facial Nerve Monitoring. World Neurosurgery 103:231240. https://doi.org/10.1016/j.wneu.2017.02.053

67. Hoshide R, Faulkner H, Teo M, Teo C (2018) Keyhole retrosigmoid approach for large vestibular schwannomas: strategies to improve outcomes. NeuroSurg Focus 44:E2. https://doi.org/10.3171/2017.11.FOCUS17607

68. Breun M, Nickl R, Perez J et al (2019) Vestibular Schwannoma Resection in a Consecutive Series of 502 Cases via the Retrosigmoid Approach: Technical Aspects, Complications, and Functional Outcome. World Neurosurgery 129:e114-e127. https://doi.org/10.1016/j.wneu.2019.05.056

69. Grinblat G, Dandinarasaiah M, Braverman I et al (2021)) "Large and giant vestibular schwannomas: overall outcomes and the factors influencing facial nerve function.. " Neurosurg Rev 44:2119-2131. https://doi.org/10.1007/s10143-020-01380-6

70. Schneider JR, Chiluwal AK, Arapi $O$ et al (2020) Near Total Versus Gross Total Resection of Large Vestibular Schwannomas: Facial Nerve Outcome. Operative Neurosurgery 19:414-421. https://doi.org/10.1093/ons/opaa056

71. Zhang Z, Nguyen Y, Seta DD et al Surgical treatment of sporadic vestibular schwannoma in a series of 1006 patients. 7

72. Starnoni D, Daniel RT, Tuleasca C et al (2018) Systematic review and meta-analysis of the technique of subtotal resection and stereotactic radiosurgery for large vestibular schwannomas: a "nervecentered" approach. NeuroSurg Focus 44:E4. https://doi.org/10.3171/2017.12.FOCUS17669

73. Carlson ML, Van Abel KM, Driscoll CL et al (2012) Magnetic resonance imaging surveillance following vestibular schwannoma resection. Laryngoscope 122:378-388. https://doi.org/10.1002/lary.22411

74. Elkashlan $\mathrm{H}$, Zeitoun $\mathrm{H}$, Arts $\mathrm{H}$ et al (2000) Recurrence of acoustic neuroma after incomplete resection. Am J Otolaryngol 21:389-392. https://doi.org/10.1016/S0196-0709(00)80049-6

75. Wu H, Sterkers J-M (2000) Translabyrinthine removal of large acoustic neuromas in young adults. Auris Nasus Larynx 27:201-205. https://doi.org/10.1016/S0385-8146(00)00057-2

\section{Figures}




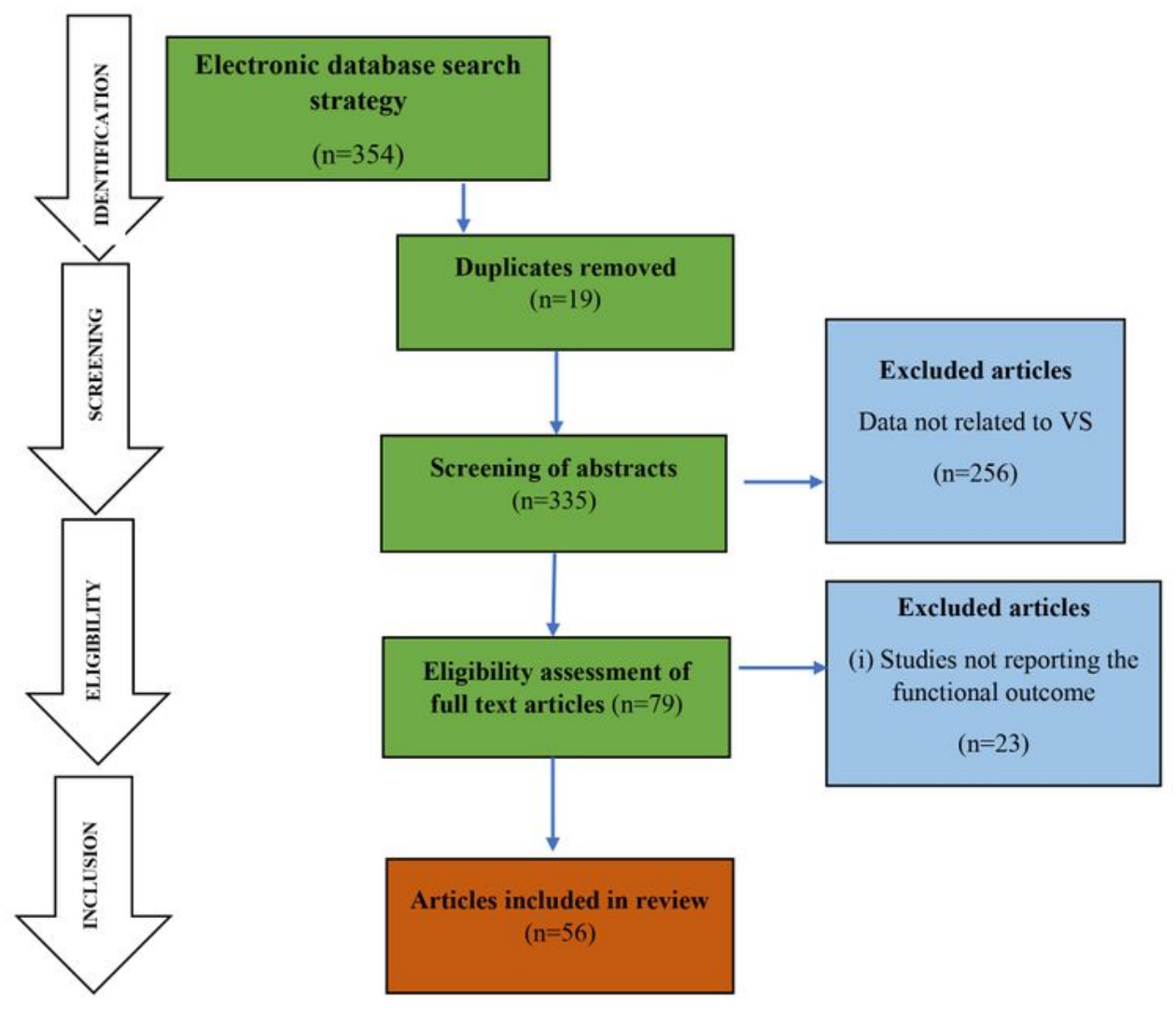

Figure 1

Electronic Database Search Strategy using PRISMA Guideline 